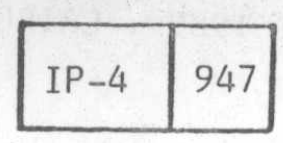

$r$
Military Technical College

CAIRO - EGYPT

\title{
APPLICATION OF IMAGE SEGMENTATION IN CLASSIFICATION OF OBJECTS
}

\author{
A.A. SHAHIN ${ }^{*}$ and H.T. KAMEL ${ }^{*}$
}

ABSTRACT

There are different methods of Image segmentation. Basically, there are two main types; parallel segmentation and sequential segmentation. In parallel segmentation, the processing that was done at each point of the picture did not depend on results already obtained at other points.

In sequential segmentation, when processing a point, advantage of the results at previously processed points can be used. In parallel approach, the same computation must be performed at every point of the picture. When using the sequential approach, simple inexpensive computations can be fulfilled to detect possible object points. Some sequential segmentation techniques are edge and curve tracking, region growing and partitioning. Partitioning of picture into regions can be constructed by starting with an initial partition and allowing both merging and splitting of regions. The initial partition may be trivial or it can be the result of previous segmentation process. The criteria for merging and splitting should depend, if possible, on how well the partition conforms to a model for the given class of pictures. In the absence of such a model one can use general purpose criteria based on such factors as region homogenity, distinctiveness of adjacent regions, edge strength, size, shape simplicity and soon just as in the case of region growing. The analysis was done for satellite imagery of location(MineaEgypt). Four classes are considered; each class is specified by its lower and upper limit of its grey level.

* Head of A/C Elect. Department, Military Technical College. Applicant (Ph.D. Study), Military Technical College. 
I- INTRODUCTION:

Segmentation of satellite images for remote sensing applications is not an easy task. It is experimently known that segmentation methods based on edge detection only are inefficient because of lack of contour information. Classification methods often provide a fragmentation of the area into numerous and sparse samples. Relaxation techniques using radiometric and spatial criteria are expensive and require subtle adjustments[1].

The method which appears most suitable for efficient segmentation of satellite images is the so called "split-and merge" method (SM method). The fundamentals of this method has long existed in the image processing literature [2].

SM method gives a direct control on the homogenity of the deduced areas. Thus it is usually pertinent.

II- Split- and Merge Algorithm:

The SM method can be summarized as follows:

Let $x$ be an image and $f(i, j)$ the radiometric value of any pixel. Let $S_{i}$ be a connected subset of $x$ and $d\left(S_{i}\right)$ any measure of heterogenity of the subset $S_{i} \cdot$ Let $P\left(S_{i}\right)$ be a logical homogenity predicate (for instance,

$P\left(S_{i}\right)=$ True iff $d\left(S_{i}\right)<\boldsymbol{\epsilon}$, with $c$ a given threshold).

The SM method provides a segmentation which ensures:
a) $\quad x=U_{i=1}^{m} S_{i}$
b) $\quad s_{i} \cap s_{j}=\varnothing$
c) $\quad P\left(S_{i}\right)=$ true, $\forall_{i}$
d) $\quad P\left(S_{i} U S_{j}\right)=$ false if $S_{i}$ and $S_{j}$ are adjacent subsets. 
Two procedures will be used:

- The merge procedure attempts to fuse a subset $S_{i}$ with all its neighbors until d criterion is met.

- The split procedure constrains array subset to verify criterion $\mathrm{C}$.

Starting with a regular partition of $\mathrm{x}$ (i.e., a square block):

- Firstly the merge procedure is applied to enlarge subsets $\mathrm{S}_{i}$, then the split procedure is applied to break the initial squares which do not satisfy C. Finally, the small blocks which verify $d$ are merged together. The Horowitz and pavlidis heterogenity measure used is: [3]

$$
d\left(S_{i}\right)=\max _{S_{i}} f(k, 1)-\min _{S_{i}} f(k, 1)
$$

Because it is fast and efficient, but any heterogenity measure could be used (for instance, variance or interquartil distance).

The picture is regularly scanned (from top to bottom and left to right) at each level $\mathrm{n}$, the question is asked whether the four subsets $\mathrm{s}_{11}^{\mathrm{n}}, \mathrm{s}_{12}^{\mathrm{n}}, \mathrm{s}_{21}^{\mathrm{n}}, \mathrm{s}_{22}^{\mathrm{n}}$ can be merged to build a homogenious set $s_{11}^{n+l}$ or not (Fig. 1). If $P\left(S_{11}^{n+1}\right)$ is true, then $S_{11}^{n+1}$ is built, and the method moves to the next blocks: $\mathrm{s}_{31}^{\mathrm{n}}, \mathrm{s}_{32}^{\mathrm{n}}, \mathrm{s}_{41}^{\mathrm{n}}, \mathrm{s}_{42}^{\mathrm{n}}$. It is clear that such a method is sequential, thus the result will be dependent on the scanning order.

III- Implementation Procedure:

The image under study is divided into square blocks with certain initial width. The merging operation starts with searching about homogeneous region produced by blocks of double initial width, the blocks are classified according to their grey level. The homogeneous blocks are merged in one block and the operation is continued until the homogenity measure is not acceptable [4]. 
This will be done for different blocks of the image, the sequence is continued with changing the level of segmentation, merging is continued and if a block do not satisfy the condition for merging, splitting is started and then returned to merging again. As shown in Fig. (2), merging is started by blocks $1,2,3$ \& 4 if they have the same class [5].

During merging opration for different image blocks, if one block is not classified to any class, the splitting operation starts. Splitting divides the block into four smaller blocks. The ranks of the splitted blocks will be complement of $\mathrm{C}$, where $\mathrm{C}$ is the total number of blocks of the whole image [6]. As shown in Fig. (3), the width of splitted blocks 2,3 \& 4 will be half the width of the unclassified block. Splitting continues until a homogeneous block is obtained. The splitting and merging process continues until all blocks are classified according to their classes. Figure (4) shows the algorithm architecture of "Splitting and Merging" [7].

IV- Application:

The splitting and Merging is applied on LANDSAT satel-lite imagery of Al-Minea Location, Egypt. The processing has been fulfilled using RIPS (Remote Image Processing system) in M.T.C. (Military Technical College) (Fig. 5). The Al-Minea image is firstly digitized (Fig. 6), then stored on a floppy disk. By using the built-in software, the digitized image can be visualized on colour monitor display. A suitable part of image (Fig. 7) is selected by the designed program and is visualized separately before applying the split and merge technique. The subimage is investigated to define the margin of grey levels for different classes [8]. These margins are fed to the Merge \& Split program before running it. After applying the preceding program, the tabulated results of classes for different image blocks are visualized as shown in Fig. (8). The same procedure is applied on the same subimage but using other segmentation levels as shown in Fig. (9) [9]. 


\section{RESULTS:}

The chosen region includes different objects (culture, town, desert, and water). The chosen window is (32 x 32), its location is centered at $(40,170)$. The level of segmentation starts with level 1 (initial block width 2). The satellite imagery of Al-Minea, Egypt (Fig. 6), the initial portion of the image is shown in Fig. (7), which is a part of the satellite image, Al-Minea. The segmented images after applying the designed program (S merge) are shown in Fig. (8) $\left(L_{S}=2\right)$ and Fig. (9) $\left(\mathrm{L}_{\mathrm{S}}=1\right)$ where we can recognize region $\mathrm{A}$ (Cultivated area) region $\mathrm{B}$ (Town and buildings), region $\mathrm{C}$ (Water), D, F (Desert). A sample of results of classification are tabulated in Table (1), where:

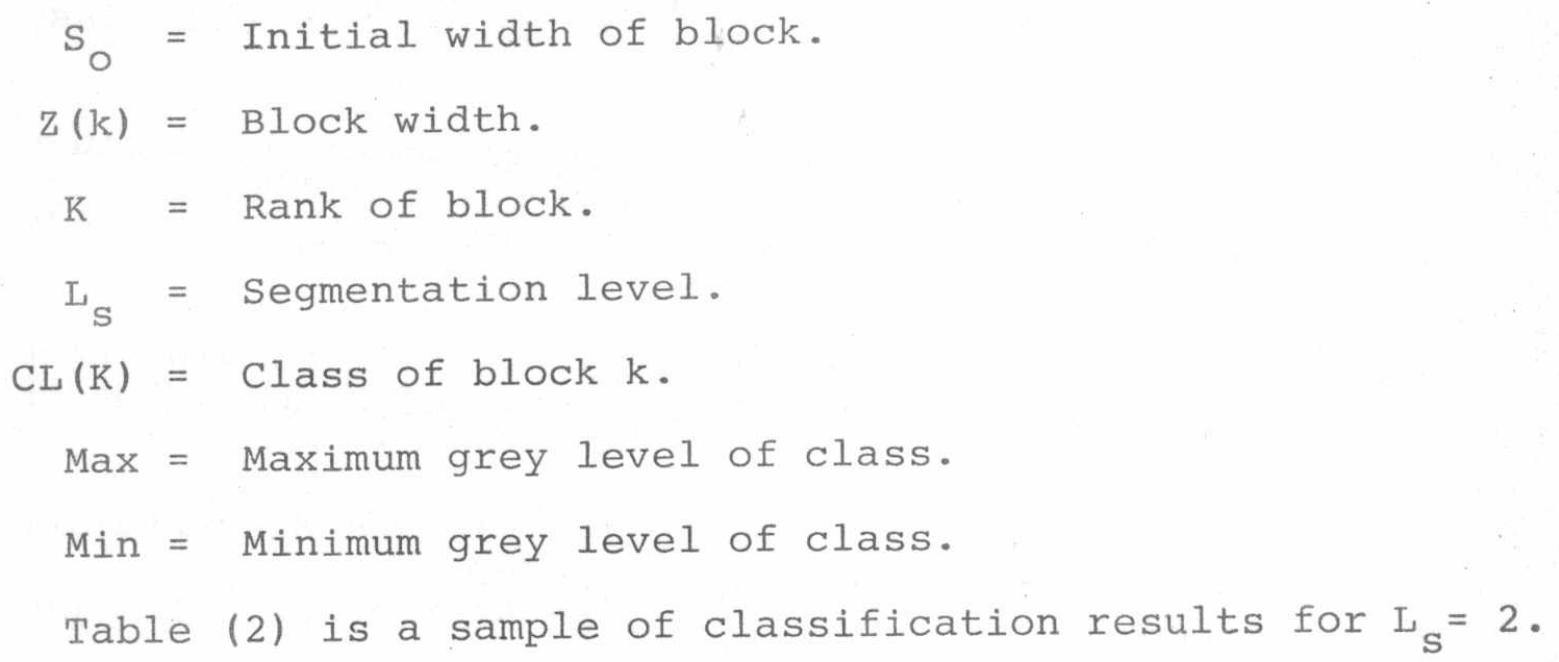

\section{VI- CONCLUSION:}

In spite of the simpler features that has been considered in our algorithm for segmentation of the satellite-image MINEA (Fig. 6), the results shown in Fig. (9) is satisfactory. The different classes, culture, river, town, and desert are clearly segmented, the different classes than the preceding four classes are not considered. This work introduced here can be continued by using another features as textural features or others. The result can be expected to be more interesting and fruitful.

Finally, this work can be used successfully with different classification methods to classify an image with high percentage of recognition. 
$\Gamma$

\section{REFERENCES:}

(1) Fusak Cheevasuvit, H.M. aitre and D.V. Madjar;

"A Robust Method for picture segmentation based on a Split-and-Merge procedure., C.V. and Image processing 1986.

(2) A. Rosennfeld, Avinash C. KAK, Digital Picture proces sing, 1982 .

(3) Horowitz and Pavlidis, picture processing by graph analysis (proc. of IEEE Conf. on Comp. Graph. 1975) Proceeding, 2nd International Joint Conference on Pattern Recognition, 1974.

(4) Kenneth R. Castleman, Digital Image Processing, 1980.

(5) W.K. Pratt, Digital Image Processing, 1978.

(6) P.C. Chen and T. Pavlidis, Image segmentation as an estimation problem, comput. Graphics Image Process, $12,1980,153-172$.

(7) P.C. Chen and T. Pavalidis, segmentation by texture using co-occurrence matrix and split and Merge algorithm, comput. Graphics, Image processing, 10, 1979, 172-182.

(8) S.L. Horowitz and T. Pavlidis, picture segmentation by a tree traverse algorithm, J. Assoc. Comput., March 23, $(1976), 368-388$.

(9) S.L. Horowitz and T. Pavlidis, Picture Segmentation by a direct split and merge procedure.

"Proceeding 2nd. Int. Conf. on Pattern Recognition Copenhagen, Aug. 1974". 
$r$

\begin{tabular}{|c|c|c|c|c|}
\hline$s_{11}^{n}$ & $s_{12}^{n}$ & $\cdots$ & & \\
\hline$s_{i 1}^{n}$ & $s_{22}^{n}$ & $\cdots$ & & \\
\hline$s_{31}^{n}$ & $s_{32}^{n}$ & $s_{33}^{n}$ & & \\
\hline$s_{41}^{n}$ &.$\cdot$ & $\cdots$ & $\cdots$ & \\
\hline
\end{tabular}

Fig. 1. Scanning of a picture during the SM procedure. $S_{i j}^{n}$ is a subset at level $n$, a candidate to be merged with its neighbors.
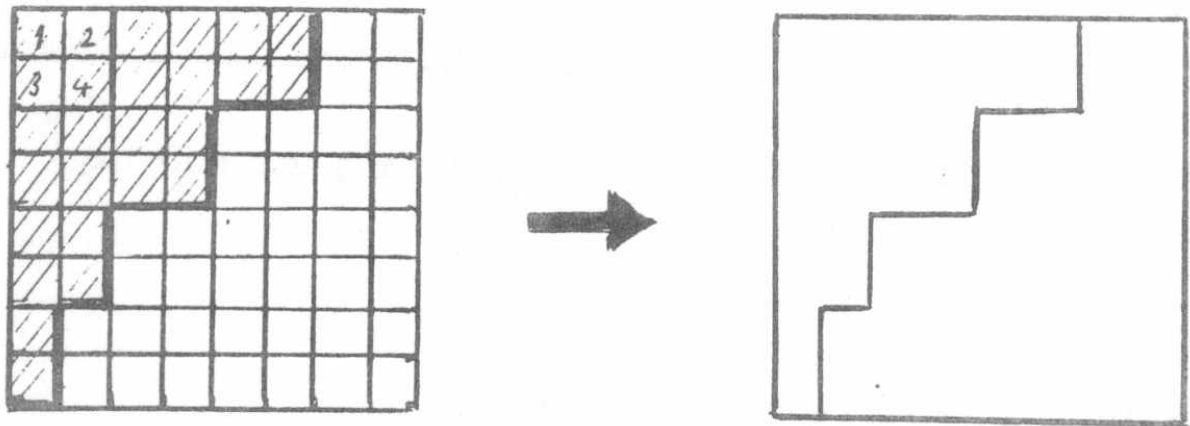

Fig. 2. Merging process
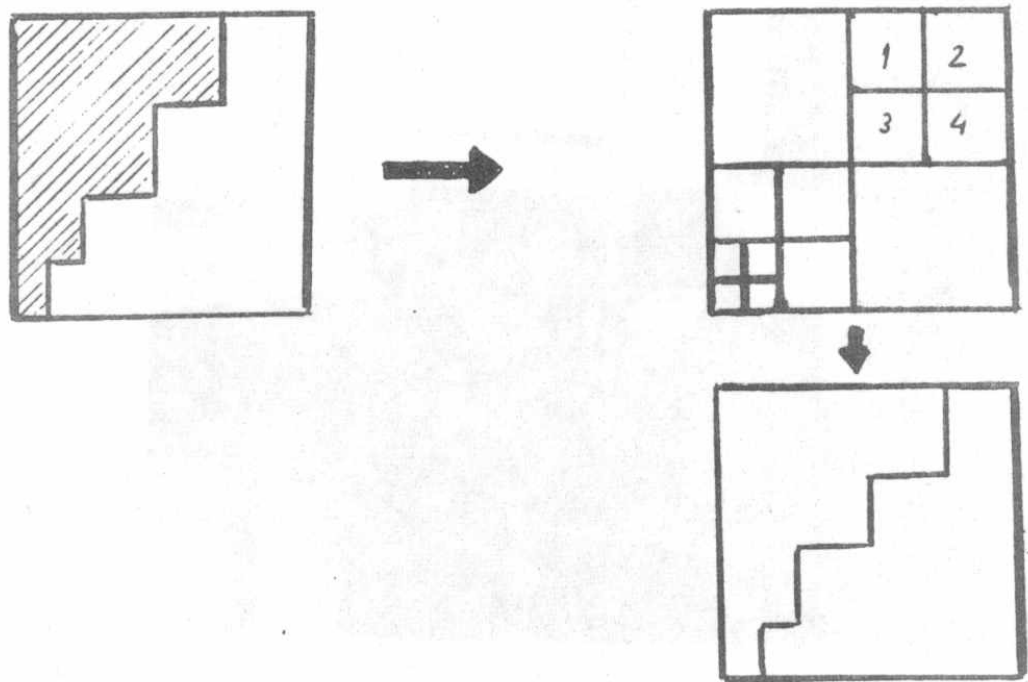

Fig. 3. Splitting Process 
Third ASAT Conference

\section{\begin{tabular}{l|l}
\hline IP-4 & 954 \\
\hline
\end{tabular}}

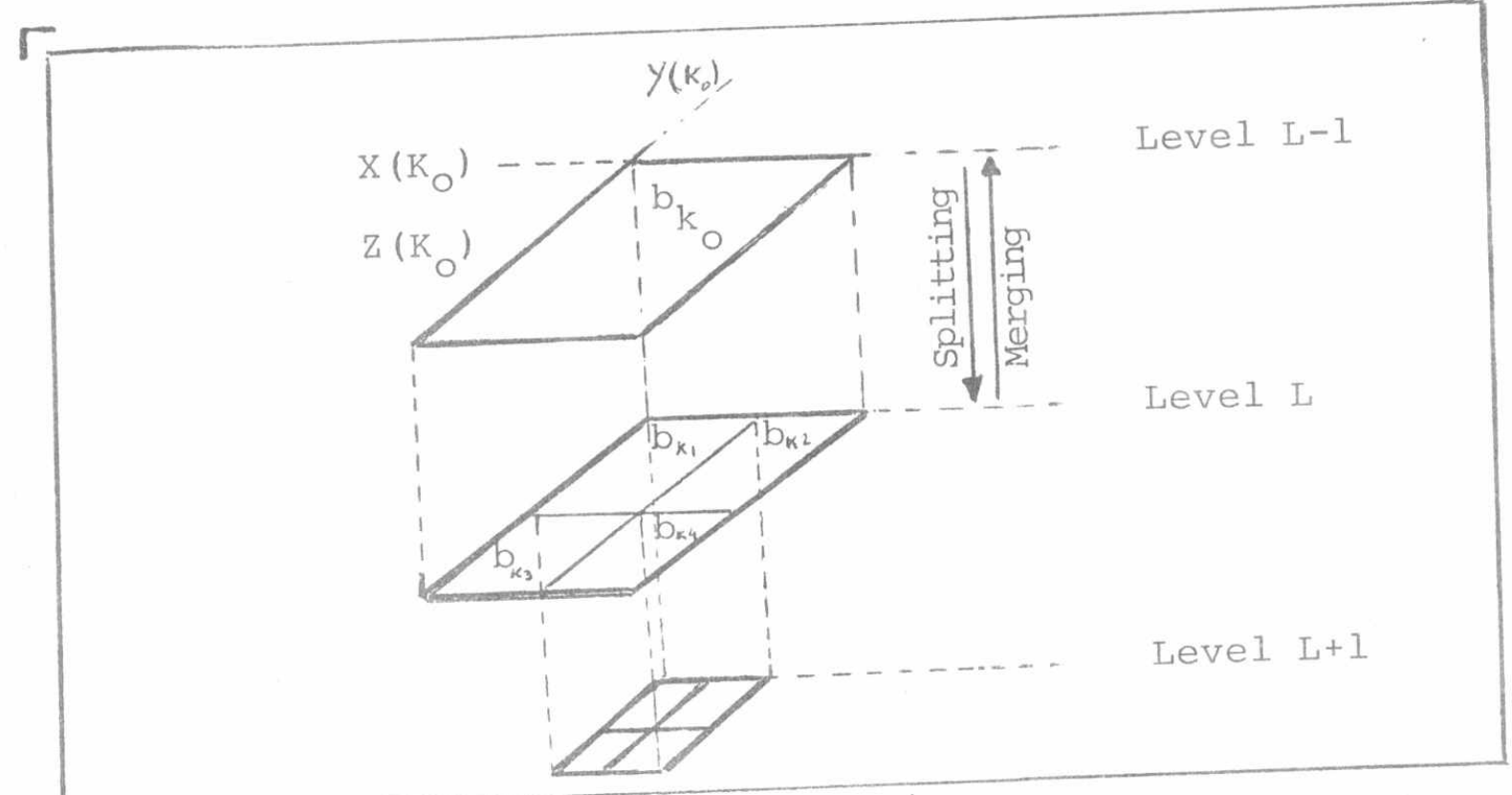

Fig. (4): Algorithm architecture of splitting and Merging.

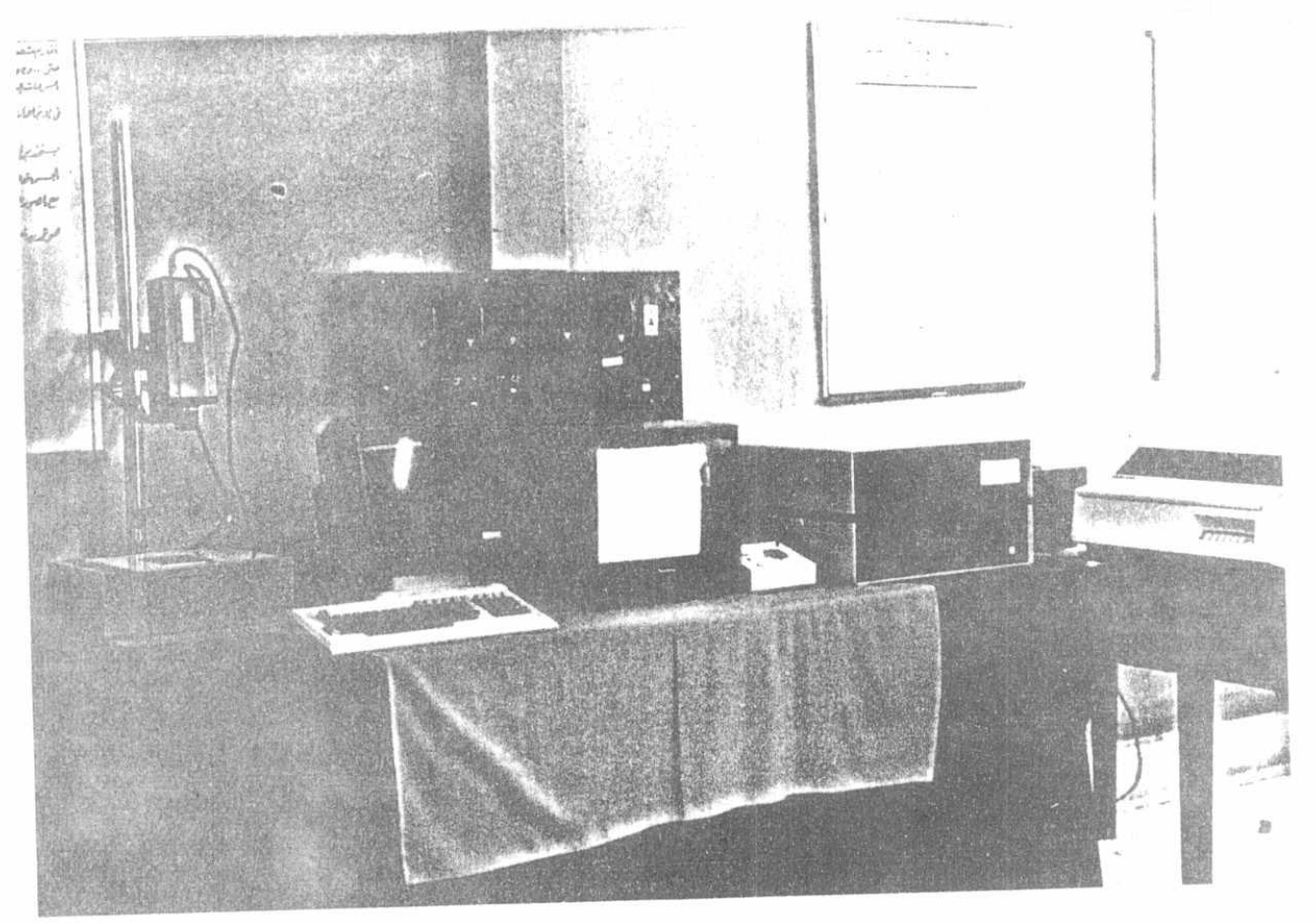

Fig. (5): Remote Image Processing System. 
\begin{tabular}{|l|l|}
\hline IP-4 & 955 \\
\hline
\end{tabular}

$r$
Third ASAT Conference

4-6 April 1989 , CAIRO

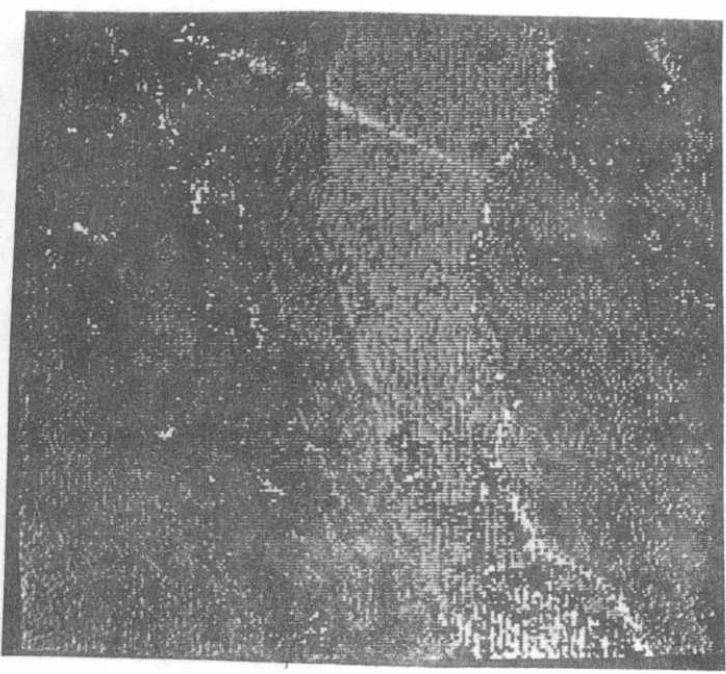

Fig. (6): Al-Minea Digitized Image.

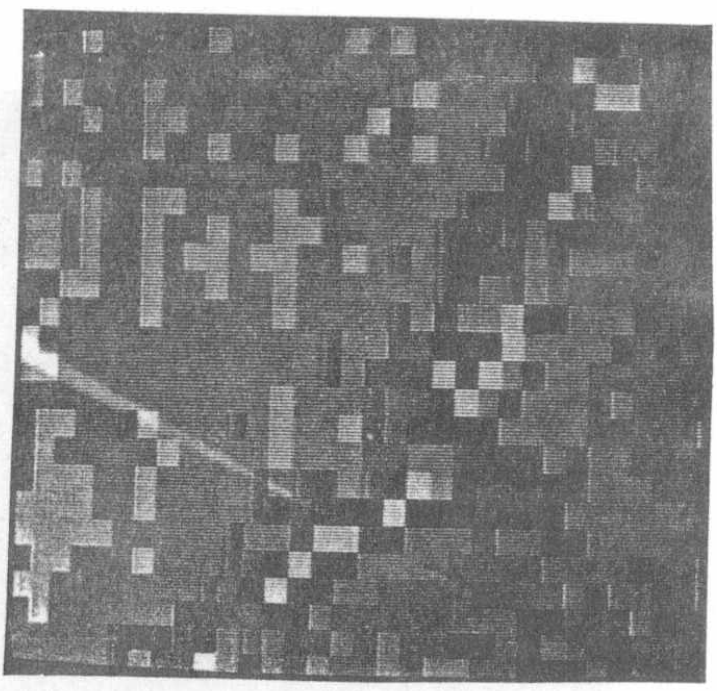

Fig. (7): $\therefore$ selected portion of Al-Minea Image $(32 \times 32)$. 


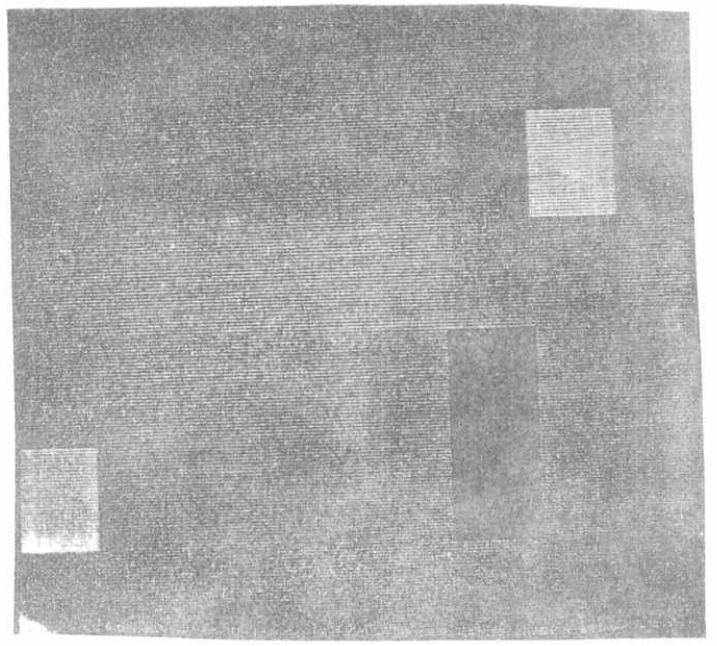

Fig. (8): The Segmented Image, $I_{S}=\mathbf{z}$.

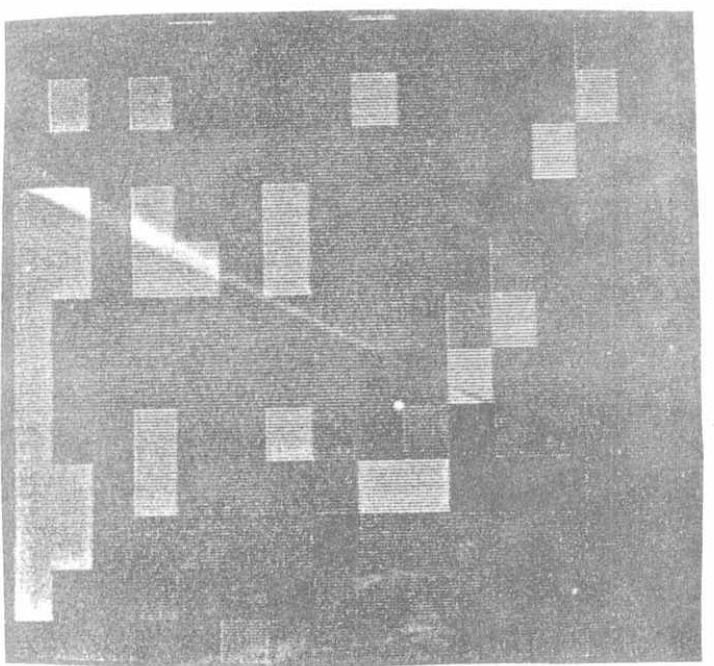

Fig. (9): The Segmented Image, $L_{S}=1$. 


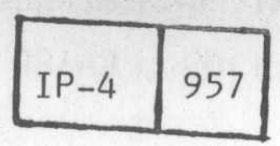

Third ASAI Conference 4-6 April 1989 , CAIRO

$r$

TABLE 1 SAMPLE OF CLASSIFICATION RESULTS

Initial number of blocks $=1024$

Initial width of block $=2$

Class 1

Class 2

Class 3

Class 4

Class 5

Class 6

$$
\mathrm{L}_{\mathrm{S}}=1
$$

$\begin{array}{ll}\text { Min }=30 & \text { Max }=51 \\ \text { Min }=52 & \text { Max }=67 \\ \text { Min }=68 & \text { Max }=95 \\ \text { Min }=96 & \text { Max }=125 \\ \text { Min }=126 & \text { Max }=140 \\ \text { Min }=141 & \text { Max }=179\end{array}$

\begin{tabular}{|c|c|c|c|c|c|c|c|}
\hline $\mathrm{K}$ & CL & $\mathrm{K}$ & CL & $\mathrm{K}$ & CL & $\mathrm{K}$ & CL \\
\hline $\begin{array}{l}91 \\
3 \\
3 \\
3 \\
9 \\
9 \\
11 \\
13 \\
13 \\
15 \\
17 \\
18 \\
19 \\
20 \\
21 \\
21 \\
22 \\
23 \\
24 \\
25 \\
23 \\
27 \\
27 \\
28 \\
29 \\
30 \\
31 \\
31 \\
65 \\
67 \\
69 \\
71 \\
73 \\
73\end{array}$ & $\begin{array}{l}5^{2} \\
5 \\
5 \\
5 \\
5 \\
5 \\
5 \\
4 \\
4 \\
4 \\
5 \\
5 \\
0 \\
4 \\
0 \\
0 \\
5 \\
0 \\
0 \\
0 \\
0 \\
5 \\
0 \\
0 \\
0 \\
0 \\
0 \\
0\end{array}$ & $\begin{array}{r}83 \\
85 \\
87 \\
89 \\
91 \\
93 \\
95 \\
129 \\
131 \\
133 \\
135 \\
137 \\
139 \\
141 \\
143 \\
145 \\
147 \\
151 \\
155 \\
157 \\
159 \\
193 \\
195 \\
197 \\
199 \\
201 \\
203 \\
205 \\
207 \\
209 \\
211 \\
213\end{array}$ & $\begin{array}{l}5 \\
5 \\
5 \\
6 \\
4 \\
0 \\
0 \\
5 \\
5 \\
5 \\
5 \\
5 \\
5 \\
5 \\
5 \\
5 \\
5 \\
5 \\
5 \\
0 \\
0 \\
5 \\
4 \\
5 \\
4 \\
5 \\
5 \\
5 \\
5 \\
4 \\
5 \\
5\end{array}$ & $\begin{array}{l}215 \\
217 \\
219 \\
221 \\
223 \\
257 \\
259 \\
261 \\
263 \\
265 \\
267 \\
269 \\
271 \\
273 \\
275 \\
277 \\
279 \\
281 \\
283 \\
285 \\
287 \\
321 \\
323 \\
225 \\
327 \\
329 \\
331 \\
333 \\
335 \\
337 \\
339 \\
341\end{array}$ & $\begin{array}{l}6 \\
6 \\
4 \\
0 \\
0 \\
5 \\
5 \\
5 \\
5 \\
5 \\
5 \\
5 \\
5 \\
5 \\
5 \\
5 \\
6 \\
4 \\
2 \\
2 \\
0 \\
4 \\
4 \\
5 \\
4 \\
5 \\
5 \\
4 \\
5 \\
5 \\
5 \\
6\end{array}$ & $\begin{array}{l}343 \\
345 \\
347 \\
349 \\
351 \\
385 \\
387 \\
391 \\
393 \\
395 \\
397 \\
399 \\
401 \\
403 \\
405 \\
407 \\
409 \\
411 \\
413 \\
415 \\
449 \\
451 \\
453 \\
455 \\
457 \\
459 \\
461 \\
463 \\
467 \\
469 \\
471 \\
473\end{array}$ & $\begin{array}{l}6 \\
3 \\
2 \\
0 \\
0 \\
4 \\
4 \\
4 \\
4 \\
5 \\
4 \\
5 \\
5 \\
5 \\
6 \\
5 \\
2 \\
2 \\
0 \\
0 \\
4 \\
5 \\
5 \\
5 \\
5 \\
5 \\
5 \\
5 \\
6 \\
5 \\
4 \\
2\end{array}$ \\
\hline
\end{tabular}




\section{TABLE 2 SAMPLE OF CLASSIFICATION RESULTS}

Initial width of block $=2$

Initial number of blocks $=1024$

$$
\mathrm{L}_{\mathrm{s}}=2
$$

$\begin{array}{ll}\text { Class } & 1 \\ \text { Class } & 2 \\ \text { Class } & 3 \\ \text { Class } & 4 \\ \text { Class } & 5 \\ \text { Class } 6\end{array}$

$$
\begin{aligned}
& \text { Min }=30 \\
& \text { Min }=52 \\
& \text { Min }=68 \\
& \text { Min }=96 \\
& \text { Min }=126 \\
& \text { Min }=141
\end{aligned}
$$

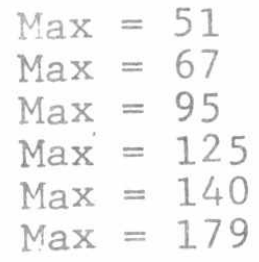

\begin{tabular}{|l|l|l|l|l|l|l|l|}
\hline $\mathrm{K}$ & $\mathrm{CL}$ & $\mathrm{K}$ & $\mathrm{CL}$ & $\mathrm{K}$ & $\mathrm{CL}$ & $\mathrm{K}$ & $\mathrm{CL}$ \\
\hline 1 & 5 & 265 & 5 & 529 & 6 & 797 & 2 \\
5 & 5 & 269 & 5 & 533 & 3 & 897 & 5 \\
9 & 5 & 273 & 5 & 537 & 2 & 901 & 6 \\
13 & 5 & 277 & 6 & 541 & 2 & 905 & 4 \\
17 & 5 & 281 & 4 & 641 & 4 & 909 & 2 \\
21 & 5 & 285 & 0 & 645 & 5 & 913 & 2 \\
25 & 6 & 385 & 5 & 649 & 5 & 917 & 2 \\
29 & 0 & 389 & 5 & 653 & 6 & 921 & 2 \\
129 & 5 & 393 & 5 & 657 & 2 & 925 & 0 \\
133 & 5 & 397 & 5 & 661 & 3 & 929 & 2 \\
137 & 5 & 401 & 5 & 665 & 2 & 931 & 4 \\
141 & 5 & 405 & 6 & 669 & 2 & 935 & 0 \\
145 & 5 & 409 & 2 & 769 & 5 & 939 & 2 \\
149 & 5 & 413 & 0 & 773 & 5 & 941 & 3 \\
153 & 6 & 513 & 5 & 777 & 6 & 945 & 6 \\
157 & 0 & 517 & 5 & 781 & 2 & 949 & 3 \\
257 & 5 & 521 & 5 & 789 & 2 & 953 & 3 \\
261 & 5 & 525 & 5 & 793 & 2 & 957 & 3 \\
& & & & & & & \\
\hline
\end{tabular}

